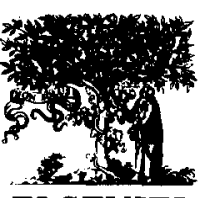

ELSEVIER

Ultrasonics $36(1998) 449-453$

\title{
The influence of inhomogeneity on the propagation of ultrasound in wood
}

\author{
F.E. Feeney ${ }^{a, *}$, R.C. Chivers ${ }^{b}$, J.A. Evertsen ${ }^{c}$, J. Keating ${ }^{d}$ \\ ${ }^{a}$ R. T.C. Carlow, Kilkenny Road, Carlow, Ireland \\ ${ }^{b}$ Department of Physics, University of Surrey, Guildford, UK \\ ${ }^{c}$ Forest Products Department, Forbairt, Glasnevin, Dublin 9, Ireland \\ ' Computer Science Department, St Patrick's College. Maynooth, County Kildare, Ireland
}

\begin{abstract}
The use of ultrasound for determining the elastic constants of materials is a well-established science for homogeneous materials such as metals. However, its extension to anisotropic, inhomogeneous materials such as wood has proved more problematic. Wood is modelled as an orthorhombic material with the influence of inhomogeneities generally being neglected. For this paper the potential influence of inhomogeneities on waves propagating in the radial direction was considered. Within ring density and ultrasonic velocity measurements were made. A model for ultrasound propagation in the radial direction was then constructed which treats the annual ring structure in the radial direction as a layered structure and predicts the occurrence of stop bands in the frequency domain. Evidence for the existence of such stop bands is considered. 01998 Elsevier Science B.V.
\end{abstract}

Keywords: Elastic components; Inhomogeneous materials; Wave propagation

\section{Introduction}

The determination of the elastic properties of a material by the measurement of the velocity of acoustic waves in the material is a long-established science. Its application to the propagation of acoustic waves in anisotropic materials is also well understood [1]. A fundamental assumption normally made when describing the propagation of acoustic waves in an anisotropic material is that the material is considered to be homogeneous along each axis of symmetry in the material. Using a wavelength an order of magnitude greater than the observed inhomogeneity is usually accepted as meeting the requirement of homogeneity.

Wood is generally modelled as an orthorhombic solid, having three mutually perpendicular axes of symmetry $[2,3]$. These three axes of symmetry are designated as follows (Fig. 1). The longitudinal axis of the tree is along the axis of the trunk or stem of the tree. The radial axis of symmetry follows a line from pith to bark of the tree. The tangential axis of symmetry is locally

\footnotetext{
* Corresponding author. Fax: + 353-503-43787;
}

e-mail: feeney@pat.rtc-carlow.ie defined as the direction perpendicular to the radial axis and the longitudinal axis of the tree.

In this paper, the propagation of acoustic waves along the radial axis of symmetry is examined. In particular, the influence of inhomogeneity due to the presence of annual rings is investigated. The annual ring structure observed in wood results from variations in the growth cycle of trees within each year. In the Spring-early Summer season, fast growth occurs and a band of lessdense early wood is laid down. In late Summer-Autumn, growth slows down and a narrow band of dense late wood is laid down. This annual growth pattern gives rise to a quasi-periodic structure of the density. The

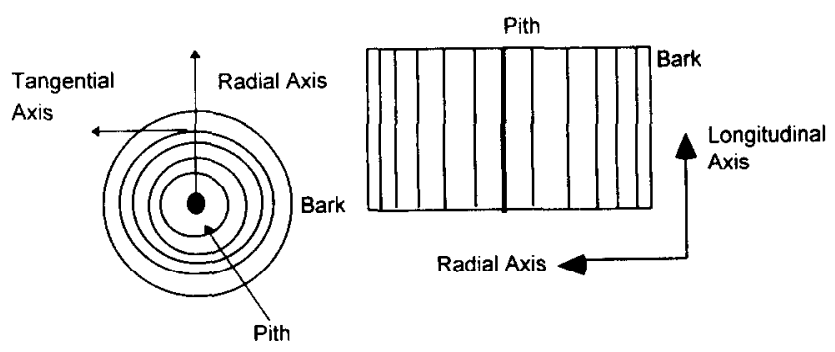

Fig. 1. Axes of elastic symmetry in wood. 
density periodicity leads, of course, to acoustic impedance and acoustic wave speed periodicities.

Models of the behaviour of periodic structures are well known with Brillouin [4] and Brekovskikh [5] having discussed their origin and implications for solidstate materials. Their application to the acoustic regime has been the subject of renewed interest $[6,7]$. The work presented here discusses the influence of the periodicity of wood in the radial direction on acoustic wave propagation in this direction. The model used is based on that first developed by Brillouin as adapted by Gazanhes and Sageloli [8]. This model provides a theoretical basis for the prediction of the existence of stop bands and pass bands at different frequencies. While the existence of a periodic array of infinite extent is not an appropriate representation of a material such as wood, James et al. [9] have shown that a finite number of layers, as little as five, can give rise to the existence of sonic band gaps.

To provide the necessary information to model wood samples as periodic structures, experimental determinations of density and velocity variations in the radial direction were carried out. These were then used to give a prediction of the likely existence of stop bands in several different regimes such as might be encountered in softwoods. Experimental evidence for the existence of stop bands was then sought.

\section{Theoretical considerations}

As described earlier, wood may be modelled as an orthorhombic solid with three mutually perpendicular axes of symmetry. Along the radial axis of symmetry, there exists a periodic variation in the material properties due to the seasonal nature of the annual growth pattern which leads to wood formation. Let us consider the propagation of a plane wave along the radial axis of a specimen of wood. First, we treat the homogenous, unbounded case. The propagation of the vector field describing the wave propagating along the axis form position $x$ to position $x+\mathrm{d} x$ may be described by the transfer matrix.

$\mathbf{T}=\left[\begin{array}{cc}\cos \frac{\omega d}{c} & -i Z \sin \frac{\omega d}{c} \\ -\frac{i}{Z} \sin \frac{\omega d}{c} & \cos \frac{\omega d}{c}\end{array}\right]$.

This matrix depends on two characteristic qualities of the medium, its impedance $z$ and the transit time, $t=$ $d / c ; c$ is the acoustic wavespeed; $d$ is the distance travelled; and $\omega$ the angular frequency of the wave. This model may now be extended to the case of propagation in a periodic medium. The medium is considered to be composed of a succession of layers of thicknesses $d_{1}$ and $d_{2}$ and of impedances $Z_{1}$ and $Z_{2}$ (Fig. 2).

Boundary conditions require continuity of acoustic field parameters such as the particle velocity and the acoustic pressure at the interface. This leads us to a description of the vector field progressing across an elementary cell of the form:

$M=\mathbf{T}\left(\frac{d_{1}}{2}, Z_{1}\right) \mathbf{T}\left(d_{2}, Z_{2}\right) \mathbf{T}\left(\frac{d_{1}}{2}, Z_{1}\right)$.

The dispersion relationship can be obtained for the trace of $M$ to be:

$$
\begin{aligned}
\cos k d= & \cos \frac{\omega d_{1}}{c_{1}} \cos \frac{\omega d_{2}}{c_{2}} \\
& -\frac{1}{2}\left(\frac{Z_{1}}{Z_{2}}+\frac{Z_{2}}{Z_{1}}\right) \sin \frac{\omega d_{1}}{c_{1}} \sin \frac{\omega d_{2}}{c_{2}}=F(\omega),
\end{aligned}
$$

where $K=\omega / c$; in this equation, $k$ is the wave number in the medium and $c$ is the apparent wave speed. From this relationship, the pass bands and stop bands may be calculated. Pass bands occur where:

$-1<F(\omega)<1$.

In Section 3, experiments to obtain the material parameters necessary for the examination of this model are reported. From these parameters the possible existence of stop bands is discussed.

\section{Experimental work}

In order to apply the layered model to the case of wood, several physical parameters had to be measured. Parameters such as ring widths, early wood width, late wood width, early wood density and late wood density were measured using an X-ray microdensitometry technique (see Section 3.1).

Also required for the model were parameters such as early wood velocity and late wood velocity in the radial direction. The practical difficulties associated with pre-

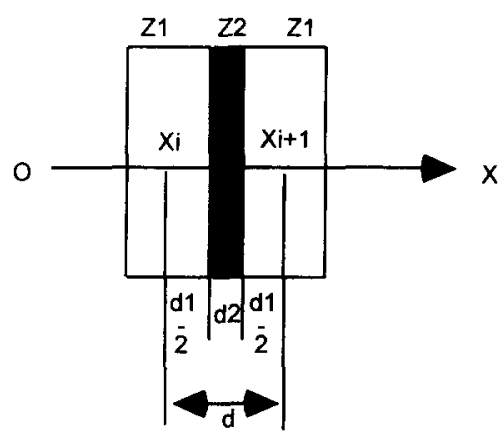

Fig. 2. Diagram of the model used to describe wave propagation in the radial direction. 
paring suitable samples for direct measurement of within-ring variations of radial velocity meant that an indirect approach was necessary to obtain these parameters. Firstly, within-ring variations of velocity in the longitudinal direction were measured by scanning a $4 \mathrm{~mm}$ disc in a water tank, as detailed in Section 3.2. Secondly, the radial velocity of a sample of wood was measured repeatedly after milling of $1 \mathrm{~mm}$ of material as detailed detailed below (Section 3.3).

\subsection{Scans of density from pith to bark}

An X-ray microdensitometer system was used to determine the variations in density in the sampled from pith to bark. An X-radiograph was scanned optically using a Joyce-Loebi microdensitometer system. The output of the densitometer was fed to a microcomputer and recorded on disk. The results of a typical density scan are shown in Fig. 3.

\subsection{Scans of longitudinal velocity from pith to bark}

In order to obtain an accurate picture of the variation of the acoustic properties of wood, from pith to bark, it was decided to make measurements of velocity in the longitudinal direction. Preparation of samples for such measurement is relatively straightforward and wood is least attenuative in this direction. Five millimeter thick discs were cut from the stems of six trees for examination.

The sample of wood under investigation was placed in a water tank in a nearly plane sound field. The field on the far side of the wood, sample to the sound source was then measured using a $0.5 \mathrm{~mm}$ hydrophone. Thus, a series of transmission measurements of the sound field throughout the sample were recorded. A special sample holder was constructed which allowed the isonifying probe and the receiving hydrophone to be held in fixed positions while the sample was moved between them. This meant that at the measuring point the incident sound field was constant. The sound source was a $2 \mathrm{MHz}$ weakly focused transducer with the hydrophone placed approximately at its focus (sce Fig. 4).

The acquired data was stored in the form of a 1000 point data file representing the averaged time trace at each measuring point. The results from a typical scan

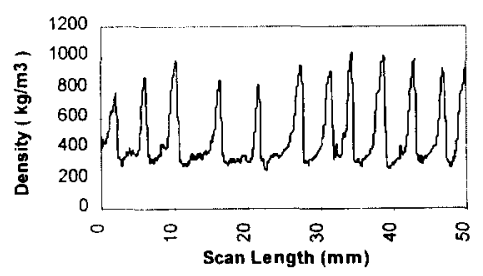

Fig. 3. Density scan from pith to bark of a typical sample used in the study.

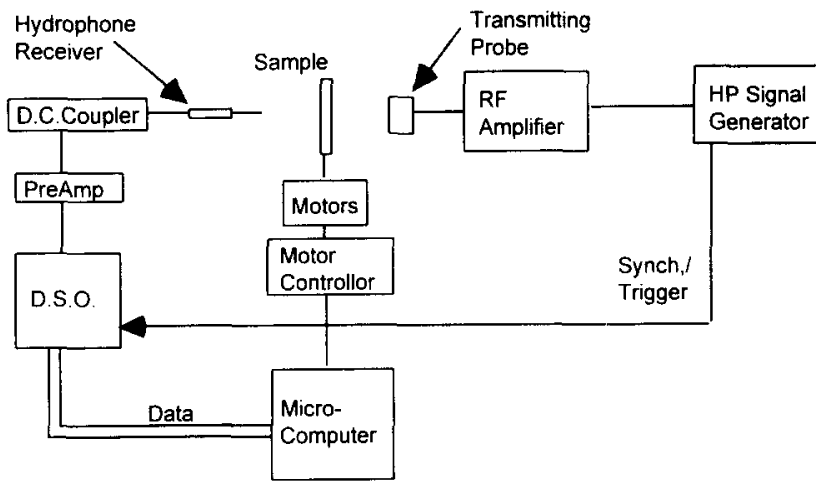

Fig. 4. Block diagram of an experimental lay-out for longitudinal velocity scans.

consisted of 201 such data files generated at $250 \mu \mathrm{m}$ intervals. This data was then combined with the data obtained from a trace obtained with no sample to calculate the ultrasonic velocity at each position in a scan. The output of a typical scan is shown in Fig. 5.

The corresponding density scan for this sample is shown in Fig. 3. It should be noted that the area sampled by the $0.5 \mathrm{~mm}$ hydrophone is much larger than the area sampled by the X-ray densitometer, leading to a reduction in sharpness of the trace. The pattern of the annual rings clearly corresponds to the pattern observed in the $\mathrm{X}$-ray sample. High velocity is observed in the late wood and low velocity in the early wood.

\subsection{Measurement of velocity variation in radial direction}

As mentioned, earlier preparation of samples suitable for direct measurement of radial velocity in early wood or late wood only is extremely difficult. It was decided instead, to try to estimate these variations by measuring average radial velocity in a sample, removing $1 \mathrm{~mm}$ of material from that sample and then remeasuring the velocity in the sample. The next step was to attribute any velocity difference to a difference in velocity in the removed layer.

There are several difficulties associated with this technique. Firstly, the very fact that the samples had to be removed from the ultrasonic measurement rig so that material could to be ground off led to variability in the results. This is because of the difficulties associated with achieving the identical coupling regime when replacing the sample in the test rig. Secondly, obtaining samples with wide rings and little distortion of the ring pattern

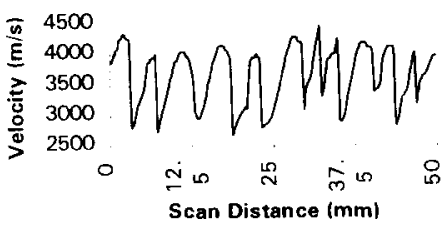

Fig. 5. Results of typical scan of velocity in the longitudinal direction. 


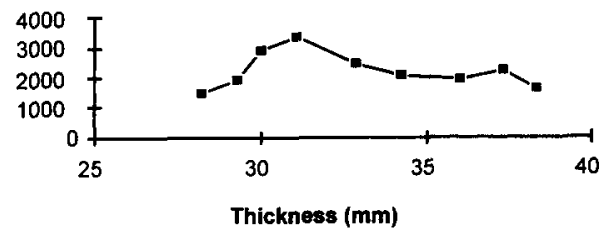

Fig. 6. Calculated velocity of a removed slice with sample thickness.

over the size of the sample block is quite difficult. Thirdly, velocity in the removed layer had to be inferred from the difference in velocity between two much largers samples of similar magnitude. Needless to say, even small experimental errors are important in such a situation.

The results of one such experiment are shown below in Fig. 6. The plot shows calculated velocities for the removed slice with decreasing sample thickness. In this plot, the effect of removing an area of late wood can be observed at around $31 \mathrm{~mm}$. The errors associated with this measurement mean that it can only be used as a guide to the actual behaviour of the sample.

\subsection{Use of experimental data in layered model}

The data obtained from the experimental study were used to provide realistic figures for Eq. (3) above. The values used are given in Table 1 and the output is given in Fig. 7. One set of values represents data from a young tree in the present study. The other represents more typical data for mature softwood. Remembering that pass bands occur for the condition that $F(\omega)$ lies in the range -1 to +1 , it is clear that for some frequencies are not allowed. However, the value of $F(\omega)$ does not climb far above or below the stop band requirement of
$-1 /+1$. Clearly, the juvenile wood case gives rise to more potential stop bands than mature wood. These steps penetrate further into the band gap region than the latewood examples, this is due to the larger density and acoustic impedance steps observed in juvenile wood among other factors.

\subsection{Evidence for the existence of stop bands}

While the calculations above indicate that juvenile wood appears to offer the best conditions for the appearance of sonic band gaps, in practice this is not the case. The model requires that the periodicity of the structure through which the wave is passing to be very regular in both dimension and property. The annual rings observed in juvenile wood, on the other hand, display significant variability in both size and density. Annual rings in juvenile wood are also significantly more curved than in adult wood.

All this can be taken to mean that adult wood samples are more likely to give rise to sonic band gaps. However, even in adult wood samples, the necessary conditions are difficult to achieve. While reductions in received signal power at certain frequencies may often be observed, it can be difficult to attribute these reductions to a particular cause. Mode conversion and difficulties in obtaining repeatable coupling also obscure the issue. In Fig. 8 are two examples of spectra obtained from softwood samples. This figure, obtained from Scots pine with narrow ( $1 \mathrm{~mm}$ ) and uniform annual rings, appears to show a stop band at above $\mathrm{l} \mathrm{MHz}$. The second, obtained from spruce, with wider and less-regular rings, does not appear to have a stop band within the range of the probes used.

Table 1

Valucs used for layered material model

\begin{tabular}{lll}
\hline Parameter & Adult wood example & Juvenile wood (measured) \\
\hline Early wood thickness & $0.5 \mathrm{~mm}$ & $4.5 \mathrm{~mm}$ \\
Early wood velocity & $1800 \mathrm{~m} / \mathrm{s}$ & $2000 \mathrm{~m} / \mathrm{s}$ (estimated) \\
Early wood density & $600 \mathrm{~kg} / \mathrm{m}^{3}$ & $400 \mathrm{~kg} / \mathrm{m}^{3}$ \\
Late wood thickness & $0.5 \mathrm{~mm}$ & $0.8 \mathrm{~mm}$ \\
Late wood velocity & $2300 \mathrm{~m} / \mathrm{s}$ & $2500 \mathrm{~m} / \mathrm{s} \mathrm{(estimated)}$ \\
Late wood density & $700 \mathrm{~kg} / \mathrm{m}^{3}$ & $750 \mathrm{~kg} / \mathrm{m}^{3}$ \\
\hline
\end{tabular}

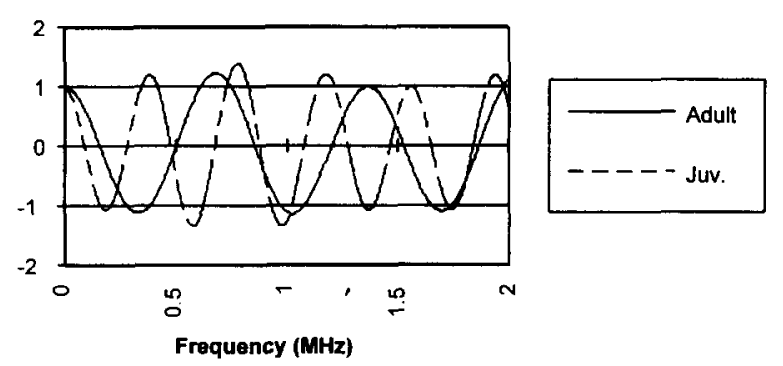

Fig. 7. $F(\omega)$ against frequency for example specimens of wood in Fig. 6 .
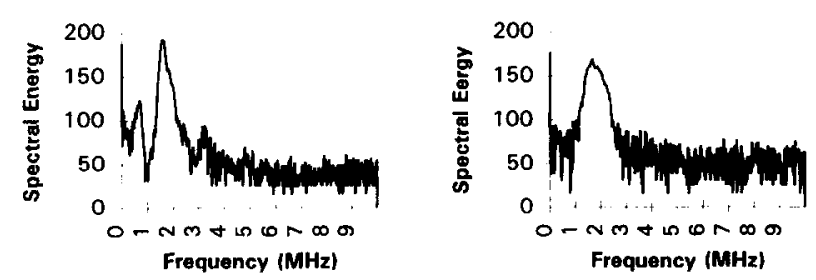

Fig. 8. Spectra of received signals from Scots pine and spruce samples. 


\section{Discussion and conclusion}

The influence of the variation in wood properties on acoustic waves due to the presence of annual rings has been considered. It has been shown that this inhomogeneity along the radial axis can have significant effects on acoustic waves of certain frequencies. Physical measurements of within ring variations in acoustic properties have been carried out. Significant variations in the acoustic properties along several axes of symmetry within a tree have been observed. In particular, a sharp impedence step between early and late wood provides a strong potential scattering source within the material along the radial axis.

The inhomogeneity due to the annual ring structure has been modelled as a periodic array. It has been shown that this may give rise to sonic band gaps under certain conditions. It is also clear that the inhomogeneity of wood along the radial direction cannot be ignored when making acoustic wave speed measurements. Even frequencies as low as $100 \mathrm{kHz}$ give rise to wavelengths of a similar order of magnitude as juvenile ring widths.

\section{References}

[1] R.E. Green, Ultrasonic investigations of Mechanical Properties, in: H. Herman (Ed.), Treatise on Materials Science and Technology, vol. 3, Academic Press, New York, 1973.

[2] M. Musgrave, Crystal Acoustics, Holden Day, San Fransisco, 1970.

[3] V. Bucur, An ultrasonic method for measuring the elastic constants of wood increment cores bored from living trees. Ultrasonics 21 (1983) 116-126.

[4] L. Brillouin, M. Parodi, Propagation des Ondes dans les Milieux Periodiques, Masson et Cie, Paris, 1956.

[5] L.M. Brekovskikh, Waves in Layered Media, Academic Press, New York, 1980.

[6] E.N. Economou, M. Sigalas, Stop bands in composites, J. Acoust. Soc. Am. 95 (4) (1995) 1734-1740.

[7] R. Esquivel-Sirvent, G.H. Cocoletzi, Band structure for the propagation of elastic waves in superlattices, J. Acoust. Soc. Am. 95 (1) (1994) 86-90.

[8] G. Gazanhes, J. Sageloli, Ftude de la dispersion acoustique de celerite dans des structures periodiques, Acustica 81 (1995) $221-227$.

[9] R. James, S.M. Woodley, C.M. Dyer, V.F. Humphrey, Sonic bands, band gaps and defect states in layered structures - theory and experiment, J. Acoust. Soc. Am. 97 (4) (1995) 20412047. 